$\Rightarrow$ BREAST CANCER

\title{
Abemaciclib effective in combination with aromatase inhibition
}

The introduction of cyclin-dependent kinase 4 and $6(\mathrm{CDK} 4 / 6)$ inhibitors has substantially improved the prognosis of women with advanced-stage breast cancer; however, the most appropriate therapy combinations and the most effective agent for each subgroup of patients have yet to be determined. Now, data from the phase III MONARCH 3 trial reveal the efficacy of abemaciclib in combination with aromatase inhibition (anastrozole or letrozole) relative to that of placebo plus aromatase inhibition in women with advancedstage hormone receptor (HR)-positive, HER2-negative breast cancer.

Patients with confirmed locoregionally recurrent or metastatic disease not amenable to surgical resection or curative radiotherapy were randomly assigned (2:1) to either abemaciclib or placebo, plus an aromatase inhibitor. Median progression-free survival (PFS) was not reached in the abemaciclib group versus 14.7 months in the placebo group (HR 0.54; $P<0.000021$ ).

Virtually all patients $(98.5 \%)$ who received abemaciclib had adverse events of any grade, with $48.6 \%$ having at least one grade $\geq 3$ adverse event. Neutropenia was the commonest grade $\geq 3$ adverse event, ocurring in $19.6 \%$ of patients. Notably, the incidence of neutropenia in this trial wassubstantially lower than that of patients with advancedstage breast cancer the PALOMA-3 trial of patients with advanced-stage breast cancer receiving a different CDK4/6 inhibitor, palbociclib, in patients with advanced-stage breast cancer in the PALOMA-3 trial; however, patients receiving abemaciclib had a substantially greater incidence of diarrhoea of any grade, including grade $\geq 3$ diarrhoea.

The findings of this study demonstrate that abemaciclib is an effective treatment of advanced-stage breast cancer, with a different adverse-event profile to that of palbociclib, although direct comparisons of the safety and efficacy of these two agents are currently unavailable.

Peter Sidaway

ORIGINAL ARTICLE Goetz, M. P. et al. MONARCH 3: abemaciclib as initial therapy for advanced breast cancer. J. Clin. Oncol. http://dx.doi.org/10.1200//CO.2017.75.6155 (2017) 